\title{
Alimentar a vida ou sustentar a morte? Uma reflexão em equipa partindo de um caso clínico
}

Sofia Esquível, ${ }^{1}$ Joana Filipa Sampaio, ${ }^{2}$ Cristiana Teixeira da Silva ${ }^{3}$

\section{RESUMO}

O uso da hidratação e nutrição artificial em doentes nos estádios finais de demência é um assunto controverso. Este tópico vai tornar-se cada vez mais importante, uma vez que a prevalência de demência vai continuar a aumentar com o envelhecimento da população e com a melhoria dos cuidados de saúde. Nos estádios mais avançados desta doença, os pacientes são incapazes de deambular ou de se alimentarem, ficando com incontinência, afasia e com importante perda da capacidade de relação. Ao serem confrontadas com um caso de demência terminal e disfagia, as autoras procuraram na literatura a melhor técnica de nutrição da doente. A decisão da inserção de uma sonda nasogástrica (SNG) ou gastrostomia percutânea endoscópica (PEG) constitui uma das mais difíceis decisões para os profissionais de saúde e familiares do idoso com demência avançada. Os estudos revistos não conseguiram comprovar que a alimentação artificial seja vantajosa na fase terminal da doença. A colocação de um tubo de alimentação (PEG ou SNG) nestes pacientes parece não acrescentar benefício para prevenir úlceras de pressão ou desnutrição e não mostrou aumento da sobrevida. Pelo contrário, esta intervenção pode diminuir a qualidade de vida por alterar a rotina de alimentação e por poder implicar a imobilização ou sedação do doente para que não retire o tubo. As diferentes técnicas de alimentação deverão ser explicadas aos familiares para os capacitar a intervir na tomada de decisão, permitindo que o doente alcance a melhor qualidade de vida possível nos seus últimos dias. São necessários mais estudos para que os riscos e benefícios da entubação de um paciente fiquem clarificados, assim como as vantagens e desvantagens de cada método.

Palavras-Chave: Demência Avançada; Sonda Nasogástrica; Desnutrição; Estado Funcional; Sobrevida.

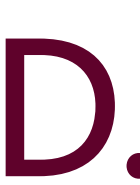

Maria, nome fictício, 77 anos, antecedentes de hipertensão arterial, dislipidemia, hiperuricemia, neoplasia do intestino com tratamento cirúrgico, insuficiência venosa, carcinoma espinocelular e demência atribuída a causa vascular e Doença de Alzheimer seguida em Psiquiatria. A D. Maria falava pouco, deambulava apenas com ajuda mas estava sempre sorridente e com fácies esboçando tranquilidade. Residiu com uma filha e frequentou um centro de dia até Abril de 2012, altura em que, por problemas familiares, foi institucionalizada num lar. A partir dessa mudança, foram sendo solicitadas visitas domiciliárias por motivos múltiplos, nomeadamente erros

\footnotetext{
1,2Interna de Medicina Geral e Familiar na Unidade de Saúde Familiar Lagoa - Unidade Local de Saúde de Matosinhos

${ }^{3}$ Assistente Graduada e Orientadora de Formação de Medicina Geral e Familiar na Unidade de Saúde Familiar Lagoa - Unidade Local de Saúde de Matosinhos
}

de administração da medicação, conflitos entre as familiares e os funcionários do lar, incumprimento de orientações médicas, cansaço, edemas dos membros inferiores... Aparentemente a D. Maria passava o dia inteiro sentada, sem incentivo à mobilização ou elevação do membro superior. Em Dezembro de 2012 foi-lhe diagnosticada Diabetes Mellitus pelo que iniciou antidiabético oral. Com a diminuição da deambulação surgiu uma úlcera de pressão na região do calcâneo direito. Continuaram a ser solicitadas visitas domiciliárias frequentes, uma vez que os cuidados de enfermagem oferecidos pelo lar se mostravam deficitários. Em Fevereiro de 2013, as filhas e funcionárias do lar notaram perda de apetite e que a doente se engasgava frequentemente. A alimentação da D. Maria era um processo difícil e moroso. A enfermeira do lar e as auxiliares de acção médica pressionaram a Médica de Família para introdução 
de uma sonda nasogástrica (SNG). Equacionou-se esta intervenção, que não foi realizada tendo em conta o desconforto causado à utente e a resistência das filhas. As mesmas consideravam que as funcionárias do lar não queriam dispender o tempo necessário com a alimentação manual. Em contexto de internamento por Infecção do Tracto Urinário (ITU), em Março de 2013, a utente teve alta com SNG colocada e foi orientada para colocação de gastrostomia percutânea (PEG). A partir dessa altura o estado geral da D. Maria deteriorou-se: ficou acamada, pouco reactiva e com os membros superiores imobilizados para que não pudesse remover a SNG, o que a deixava agitada. As filhas queixaram-se de que ficava com as mãos edemaciadas, «devido à imobilização incorrecta realizada pelas funcionárias do lar». No entanto, nesta fase já consideravam que a D. Maria tinha de ser alimentada da forma que fosse possivel, mesmo que por uma SNG. Entretanto, novo internamento para colocação da PEG. Durante a endoscopia foi descoberto um adenocarcinoma gástrico ulcerado, sem indicação cirúrgica, pelo que a PEG não foi introduzida. A utente manteve-se acamada, com SNG, imobilizada, apática, sorriso mais escasso. Foram surgindo intercorrências comuns na sua situação clínica, tendo sido minimizados os procedimentos potencialmente desconfortáveis, como a pesquisa de glicemia capilar. Foi referenciada para a equipa de Cuidados Paliativos, para otimização das medidas de conforto, mas antes da intervenção desta foi internada por nova ITU e faleceu.

Após este caso, a equipa médica que assistiu a D. Maria sentiu necessidade de reflectir sobre a orientação dos cuidados prestados e sobre a postura demonstrada perante as pressões das familiares e funcionárias do lar. Foi revista literatura com o objectivo de perceber o que poderia ser melhorado e se o desconforto a que a doente foi exposta em busca da manutenção da nutrição fez sentido no contexto da sua doença crónica avançada e terminal.

Com o envelhecimento da população tem ocorrido um aumento dramático da prevalência de demência. ${ }^{1}$ Esta patologia constitui uma síndrome neurodegenerativa progressiva com várias etiologias, sendo a causa mais comum a Doença de Alzheimer, seguida pela demência vascular e pela demência dos corpos de Lewy. ${ }^{2}$ Nos estádios mais avançados desta doença, os pacientes tipicamente estão incapazes de deambular ou de se alimenta- rem, ficando com incontinência, afasia e com importante perda da capacidade de relação. ${ }^{1}$ Perante um doente demenciado que necessite de uma cirurgia, hospitalização, medicação intravenosa ou entubação a opinião dos familiares é muitas vezes determinante na decisão. ${ }^{3}$

\section{ALIMENTAÇÃO, SÍMBOLO DE CUIDADO}

A comida e a alimentação desempenham um papel social, religioso, biológico e simbólico na maioria das culturas. ${ }^{4}$ A decisão da inserção de uma SNG ou PEG constitui uma das mais difíceis decisões para os profissionais de saúde e familiares do idoso com demência avançada. Talvez esta questão suscite mais dúvidas do que a decisão relativa à reanimação cardio-respiratória ou até à ventilação mecânica. ${ }^{1,3}$ Os profissionais de saúde são chamados a participar de uma decisão tendo em conta os seus valores, conhecimentos técnicos e ética e que deverá, idealmente, reflectir a decisão do paciente. Um estudo prospectivo recente, cujo objectivo era perceber as expectativas dos clínicos relativamente aos benefícios da introdução de um tubo de alimentação, concluiu que estes têm expectativas de ganhos significativos deste procedimento, que não se repercutem na realidade. Se os médicos comunicam estas expectativas positivas aos pacientes e familiares podem levar ao aumento do uso desta prática. Logo, é necessário maior conhecimento científico e apoio na decisão relativamente às opções de alimentação assistida, a fim de mudar este padrão de actuação médica. ${ }^{5}$ Os métodos de alimentação por SNG/PEG têm sido promovidos na comunidade como benéficos para o estado nutricional, integridade da pele, prevenção de pneumonia aspirativa, entre outros. Está subjacente a ideia de que pode melhorar o estado funcional e potencialmente aumentar a sobrevida. ${ }^{6}$ A nutrição e hidratação artificiais têm sido consideradas uma demonstração de cuidado e carinho, enquanto renunciar a estas medidas tem sido equiparado a negligência e abandono do doente. $^{7}$

\section{UMA REALIDADE CADA VEZ MAIS PRÓXIMA}

Nos lares geriátricos esta problemática tem-se tornado frequente, dada a probabilidade aumentada dos pacientes com demência avançada apresentarem problemas de deglutição e alimentação. Estes podem incluir indiferença ou recusa alimentar ou a incapacida- 
de de deglutir o bolo alimentar corretamente., ${ }^{8,9}$ Estima-se que nos lares de idosos a prevalência de disfagia ronde os $50-75 \%$, com cerca de $50 \%$ destes pacientes a apresentarem aspiração de alimentos e um terço a desenvolver pneumonia. ${ }^{10}$ Nos EUA, calcula-se que $34 \%$ dos pacientes residentes em lares, com alterações cognitivas avançadas, possuem um tubo para alimentação. ${ }^{11}$

Nos pacientes com demência as principais causas de perda de peso correspondem ao aporte nutricional insuficiente para as necessidades metabólicas, disfagia e diminuição do apetite. Na doença de Alzheimer a disfagia pode ocorrer cedo no processo de doença, não constituindo necessariamente um sintoma terminal. ${ }^{12}$ É frequente o recurso a parâmetros analíticos do status nutricional, como a albumina, hematócrito ou colesterol para justificar a colocação de um tubo de alimentação. ${ }^{13,14}$ No entanto, a desnutrição persistente em doentes cronicamente alimentados por PEG, mesmo com quantidades adequadas de fórmula, sugere que os efeitos das doenças crónicas, a imobilidade e os defeitos neurológicos podem limitar o benefício do suporte nutricional a longo prazo. ${ }^{15}$ Segundo a teoria metabólica, os indivíduos que alcançam uma fase avançada da demência têm uma taxa metabólica baixa, devido à perda de massa muscular e à atrofia progressiva da sua massa corporal magra e cérebro; o seu metabolismo basal é baixo porque são fisicamente inactivos. Para além disto, estes doentes vão perdendo peso gradualmente, o que permite que o corpo se adapte, diminuindo o seu metabolismo e retendo proteínas da dieta de forma mais eficiente. Este estado de adaptação pode persistir indefinidamente. ${ }^{16}$ Estes doentes podem ingerir menos alimentos do que parece adequado, mas, na maioria dos casos, «não estão a passar fome». Estão num estado de homeostase com baixo consumo de energia, sendo que se o peso se for mantendo constante pode não ser necessária alimentação por sonda. ${ }^{7}$

\section{A DECISÃO DE INSERIR UMTUBO DE ALIMENTAÇÃO}

A decisão da inserção de um tubo de alimentação num paciente com demência avançada está frequentemente associada a pressão por parte dos familiares e/ou dos funcionários dos lares, quando não têm o tempo necessário para a alimentação por via oral. A introdução de um tubo de alimentação é também frequen- te em contexto de internamento hospitalar com o objectivo de reduzir a duração do internamento e respectivos custos. ${ }^{3}$

A colocação de SNG tem sido associada a colonização orofaríngea patogénica significativa, quando comparada com a alimentação oral na população idosa. ${ }^{17}$ Após a realimentação, pode ocorrer uma síndrome severa com desequilíbrios hidroelectrolíticos. Nos pacientes com demência avançada é comum ocorrer estase gástrica, o que também aumenta o risco de aspiração na presença de uma SNG. Este método não mostrou aumentar a sobrevida dos pacientes. ${ }^{12}$

A colocação de uma gastrostomia percutânea é frequentemente requisitada nestes pacientes que se apresentam com dificuldade de alimentação. A PEG está associada a uma taxa de mortalidade geralmente baixa, variando de 0 a $2 \% .{ }^{3}$ No entanto, as taxas de complicações da sua inserção podem variar de 15 a 70\%, sendo o vazamento do tubo, celulite, refluxo gastroesofágico e diarreia algumas das possíveis consequências, podendo causar extremo desconforto ao paciente. ${ }^{18} \mathrm{~A}$ colocação de uma PEG apresentou em 4,3\% dos casos complicações com desfecho fatal, por abcesso intraabdominal e sépsis. ${ }^{19}$ A PEG é referida como potencialmente redutora do número de hospitalizações, assim como é enfatizada a possibilidade de administração de medicações por esta via. ${ }^{12} \mathrm{~A}$ colocação de uma PEG não significa necessariamente o fim do prazer pela comida, sendo que a via oral pode e deve ser incentivada, tendo em conta a vontade e capacidade de deglutição do paciente..$^{12}$ No entanto, devido ao facto dos doentes com demência apresentarem diarreia frequente, o statusnutricional pode não melhorar significativamente. Por outro lado, a PEG não previne a pneumonia de aspiração, sendo que este risco se mantém devido ao refluxo gástrico e aspiração de saliva. ${ }^{1}$ A diferença entre a sobrevida média dos pacientes que colocaram PEG e os que o não fizeram não foi estatisticamente significativa. ${ }^{19}$

A colocação de um tubo de alimentação pode significar também uma perda de autonomia. ${ }^{711}$ Doentes com demência e que preservem mobilidade nos membros superiores têm necessidade de ser imobilizados uma grande parte do tempo para que não retirem o tubo. A imobilização do paciente pode agravar as úlceras de pressão, para além das questões éticas que levanta, como a dignidade no fim de vida. ${ }^{1}$ Estudos mostram 
que $71 \%$ dos pacientes com demência avançada que recebem um tubo de alimentação têm de ser imobilizados. ${ }^{1}$ Também é frequente estes pacientes ficarem agitados perante o desconforto do tubo, levando a que recebam maior sedação para controlar a agitação. ${ }^{2} \mathrm{~A}$ nutrição artificial pode levar ao aumento de incontinência fecal e urinária e ao aumento das secreções pulmonares, agravando ainda mais o quadro clínico. ${ }^{2}$

Pacientes com demência avançada frequentemente perdem a sensibilidade à sede e fome, pelo que o consumo forçado de alimentos/fluidos pode causar incómodo. Oferecer alimentos familiares e fluidos em pequenas quantidades será, provavelmente, o mais adequado. ${ }^{2,20}$

A colocação de um tubo de alimentação deve ser, assim, reservada para situações específicas em que os benefícios garantidamente superam os riscos. ${ }^{7} \mathrm{Tem}$ sido muito difícil comprovar que a sua colocação prolongue a vida. Em Espanha, um estudo observacional recente, incidindo em idosos com demência avançada residentes na comunidade, identificou uma associação estatisticamente significativa entre a utilização permanente de SNG e mortalidade precoce. ${ }^{21}$ Uma revisão da Cochrane, de 2009, mostrou que a alimentação por tubo (PEG ou SNG) nestes pacientes não é benéfica a prevenir úlceras de pressão e desnutrição e a prolongar a sobrevivência. A alimentação por tubo pode diminuir a qualidade de vida por romper as rotinas de alimentação do paciente. ${ }^{2}$

Acredita-se que a alimentação manual permite ao indivíduo disfrutar da componente social de comer e assim aumentar a qualidade de vida. ${ }^{7}$ A American Geriatrics Society lançou um consenso em 2013, reforçando a não recomendação da colocação de uma PEG em idosos com demência avançada. Esta sociedade considera que a alimentação manual cuidadosa deverá ser privilegiada, pois a alimentação por tubo não apresenta melhores outcomes no que diz respeito à sobrevida, pneumonia de aspiração, status funcional e desconforto, podendo aumentar a agitação e consequentemente o uso de sedativos, obrigatoriedade de imobilização do paciente e agravamento de úlceras. Considera que devem ser feitos esforços no sentido de alterar o ambiente que envolve a refeição do idoso e procurar medidas centradas na resolução dos problemas específicos do paciente. A abordagem deve ser idealmente multidisciplinar, no sentido de encontrar causas reversíveis que possam estar a dificultar a alimentação. ${ }^{4} \mathrm{Mo}-$ dificar a textura da comida, assegurar que o doente é sentado corretamente para as refeições e obter ajuda dos familiares pode contribuir para o sucesso. ${ }^{10} \mathrm{~A}$ cavidade oral do paciente deve ser rotineiramente inspeccionada durante e após as refeições para minimizar bolsas de alimentos, ${ }^{24}$ enquanto o excesso de secreções orais pode ser aspirada para minimizar a aspiração de secreções orais contaminadas. ${ }^{23}$

Para obter evidência mais sólida seriam necessários estudos prospectivos, randomizados e controlados, o que levanta diversas questões éticas. Por esse motivo, a maioria dos estudos utilizou meios indirectos de aferição, o que enfraquece a consistência dos achados encontrados. Mesmo entre os profissionais de saúde existe uma grande heterogeneidade de prática clínica e muitas vezes não existe diálogo suficiente entre a equipa de saúde assistente e os familiares do paciente. ${ }^{3}$

\section{O PAPEL DO MÉDICO DE FAMÍLIA, AS DIRECTIVAS AVANÇADAS DE VONTADE E A REALIDADE EM PORTUGAL}

O médico de família irá deparar-se cada vez mais com casos semelhantes devido ao crescente envelhecimento da população. Para além disso, a responsabilização que a sociedade incute aos profissionais de saúde neste tipo de decisões acaba por ser confortável para os familiares. A colocação de um tubo para alimentação deve ser discutida com todos os intervenientes e apenas deverá ser realizada após estes compreenderem os riscos e benefícios, bem como ser ponderado o conforto, o risco de aspiração, a prevenção de úlceras de pressão e a sobrevida. ${ }^{19}$ As famílias terão uma maior compreensão sobre a progressão da doença se forem informadas do prognóstico. Estudos mostram que as famílias com conhecimento do prognóstico fundamentado destes pacientes apresentam menos probabilidade de quererem medidas invasivas e intervenções que possam causar desconforto. ${ }^{20}$

As Directivas Antecipadas de Vontade (DAV), nomeadamente sob a forma de testamento vital, que tanto têm sido debatidas, poderão vir a ajudar na decisão de não intervir, se for tomada em consideração que essa foi a vontade do doente quando se encontrava em plena capacidade de deliberação. 
As DAV definem-se como o acto pessoal, unilateral e livremente revogável, no qual uma pessoa maior de idade e com plena capacidade de exercício de direitos, manifesta, através de documento escrito, a sua vontade séria, livre e esclarecida no que respeita aos cuidados de saúde que deseja receber no futuro, no caso de, por qualquer causa, se encontrar incapaz de a expressar pessoal e autonomamente. Permitem também a nomeação de um Procurador de Cuidados de Saúde, pessoa a quem são atribuídos, com o devido consentimento do doente, poderes representativos em matéria de cuidados de saúde e que devem ser exercidos apenas quando o doente se encontre incapaz de manifestar a sua vontade autonomamente..$^{24}$

Alguns estudos sugerem que as DAV podem ajudar os cuidadores a fazer escolhas que melhor reflictam os verdadeiros desejos dos indivíduos, pelo que têm sido feitos esforços para aumentar a utilização destes documentos. As DAV são de particular importância para as pessoas com demência, uma vez que se trata de patologias progressivas com evolução inexorável para um estado em que já não podem comunicar os seus desejos de tratamento. Ficam, então, dependentes de cuidadores, familiares e médicos para expressar ou tomar decisões de saúde que incluem o uso de tratamentos de sustentação da vida. ${ }^{25}$

Diferentes regimes legislativos, culturas, crenças e religiões podem influenciar a heterogeneidade das práticas clínicas entre diferentes regiões do mundo. No entanto, a Alzheimer's Disease International considerou a demência como uma prioridade de saúde a nível mundial e recomendou aos governos o desenvolvimento de estratégias de âmbito nacional que prestem serviços e apoio a pessoas com demência e às suas famílias. ${ }^{26} \mathrm{Nes}-$ te sentido, seria pertinente a criação de um plano de cuidados para doentes com demência a nível nacional, que permitisse assegurar cobertura alargada e equidade do acesso, independentemente da idade, sexo, saúde, incapacidade e residência em meio rural ou urbano. ${ }^{26}$

Em suma, existe uma extrema necessidade de debater esta temática entre profissionais de saúde e informar os familiares de forma mais completa para os capacitar a intervir na decisão, tendo em conta uma melhor qualidade de vida nos últimos dias do paciente. São necessários mais estudos para que os riscos e benefícios da entubação de um paciente fiquem clarificados, assim como as vantagens e desvantagens de cada método.

\section{REFERÊNCIAS BIBLIOGRÁFICAS}

1. Gillick MR. Rethinking the role of tube feeding in patients with advanced dementia. n Engl J Med 2000 Jan 20; 342 (3): 206-10.

2. Sampson EL, Candy B, Jones L. Enteral tube feeding for older people with advanced dementia. Cochrane Database Syst Rev 2009 Apr 15; (2): CD007209.

3. Span P. When demented patients receive feeding tubes. NYTimes, 09/05/2011.

4. American Geriatrics Society. Feeding tubes in advanced dementia position statement. Disponível em: http://www.americangeriatrics.org/files/documents/feeding.tubes.advanced.dementia.pdf [acedido em 08/10/2013].

5. Hanson LC, Garrett JM, Lewis C, Phifer N, Jackman A, Carey TS. Physicians' expectations of benefit from tube feeding. J Palliat Med 2008 Oct; 11 (8): 1130-4.

6. Hanson LC, Ersek M, Gilliam R, Carey TS. Oral feeding options for people with dementia: a systematic review. J Am Geriatr Soc 2011 Mar; 59 (3): 463-72.

7. Li l. Feeding tubes in patients with severe dementia. Am Fam Physician 2002 Apr 15; 65 (8): 1605-10.

8. Finucane TE, Christmas C, Travis K. Tube feeding in patients with advanced dementia: a review of evidence. JAMA 1999 Oct 13; 282 (14): 1365-70.

9. Finucane TE. Tube feeding in the demented elderly: a review of the evidence. American Geriatrics Society Annual Scientific Meeting 2001. New York, NY: American Geriatrics Society; 2001.

10. Gallagher R., Swallowing difficulties: a prognostic report. Can Fam Physician 2011 Dec; 57 (12): 1407-9.

11. Mitchell SL, Teno JM, Roy J, Kabumoto G, Mor V. Clinical and organizational factors associated with feeding tube use among nursing home residents with advanced cognitive impairment. JAMA 2003 Jul 2; 290 (1): 73-80.

12. Regnard C, et al. Gastrostomies in dementia: bad practice or bad evidence? Age Ageing 2010 May; 39 (3): 282-4.

13. Ciocon JO, Silverstone FA, Graver LM, Foley CJ. Tube feedings in elderly patients. Indications, benefits, and complications. Arch Intern Med 1988 Feb; 148 (2): 429-33.

14. Kaw M, Sekas G. Long-term follow-up of consequences of percutaneous endoscopic gastrostomy (PEG) tubes in nursing home patients. Dig Dis Sci 1994 Apr; 39 (4): 738-43.

15. Henderson CT, Trumbore LS, Mobarhan S, Benya R, Miles TP. Prolonged tube feeding in long-term care: nutritional status and clinical outcomes. J Am Coll Nutr 1992 Jun; 11 (3): 309-25.

16. Hoffer $\mathrm{LJ}$. Tube feeding in advanced dementia: the metabolic perspective. BMJ 2006 Dec 9; 333 (7580): 1214-5.

17. Leibovitz A, Plotnikov G, Habot B, Rosenberg M, Segal R. Pathogenic colonization of oral flora in frail elderly patients fed by nasogastric tube or percutaneous enterogastric tube. J Gerontol A Biol Sci Med Sci 2003 Jan; 58 (1): 52-5.

18. Finucane TE, Bynum JP. Use of tube feeding to prevent aspiration pneu- 
monia. Lancet 1996 Nov 23; 348 (9039): 1421-4.

19. Murphy LM, Lipman TO. Percutaneous endoscopic gastrostomy does not prolong survival in patients with dementia. Arch Intern Med 2003 Jun 9; 163 (11): 1351-3.

20. Mclnttyre L, et al. End Stage dementia and palliative care, BCMJ 2013 Jul-Aug; 55 (6): 287-91.

21. Álvarez-Fernández B, García-Ordoñez MA, Martínez-Manzanares C, Gómez-Huelgas R. Survival of a cohort of elderly patients with advanced dementia: nasogastric tube feeding as a risk factor for mortality. Int J Geriatr Psychiatry 2005 Apr; 20 (4): 363-70.

22. Palmer JL, Metheny NA. Preventing aspiration in older adults with dysphagia. Am J Nurs 2008 Feb; 108 (2): 40-8.

23. Langmore SE, Terpenning MS, Schork A, Chen Y, Murray JT, Lopatin D, et al. Predictors of aspiration pneumonia: how important is dysphagia? Dysphagia 1998 Spring; 13 (2): 69-81.

24. Lei n. ${ }^{\circ}$ 25/2012, de 16 de julho. Diário da República, 1 a $^{\text {a }}$ série - N. ${ }^{\circ} 136$.

25. Triplett PM, Black BS, Phillips H, Richardson Fahrendorf S, Schwartz J,
Angelino AF, et al. Content of advance directives for individuals with advanced dementia. J Aging Health 2008 Aug; 20 (5): 583-96.

26. Alzheimer's Disease International. Relatório sobre a doença de Alzheimer no mundo - 2009: Resumo Executivo. Disponível em: http://www.alz.co.uk/research/files/WorldAlzheimerReport-Portuguese.pdf [acedido em 08/10/2013].

\section{CONFLITOS DE INTERESSE}

As autoras declaram não ter conflito de interesses.

\section{ENDEREÇO PARA CORRESPONDÊNCIA}

Sofia Esquível

Unidade de Saúde Familiar Lagoa, Rua da Lagoa, 4460-352 Senhora da Hora

E-mail: sofia.esquivel@gmail.com

Recebido em 11-10-2013

Aceite para publicação em 12-02-2014

\section{ABSTRACT}

\section{TUBE FEEDING TO SUSTAIN LIFE OR PROLONG DYING: A TEAM REFLECTION ON A CLINICAL CASE}

The use of artificial nutrition and hydration in patients in the late stages of dementia is controversial. The prevalence of dementia will continue to increase with the aging population so this topic will become increasingly important. In the more advanced stages of dementia, patients typically are unable to walk or feed themselves, becoming incontinent and aphasic. When confronted with a patient with terminal dementia and dysphagia, the authors searched the literature for the best approach to nutrition for the patient. The decision to insert a nasogastric tube or a percutaneous endoscopic gastrostomy (PEG) for elderly patients with advanced dementia is one of the hardest decisions for health professionals and relatives. The studies reviewed have failed to show that artificial feeding is advantageous in the terminal stage of the disease. They concluded that feeding tube placement in these patients is not helpful for the prevention of pressure ulcers, malnutrition, or for prolonging survival. This procedure may reduce quality of life because of the change in feeding routines. It may require immobilization or sedation of the patient to prevent them from removing the tube. Family members should be more informed to make better decisions to improve the quality of life in the last days of the patient. Further studies are needed so that the risks and benefits of tube feeding are clarified as well as the advantages and disadvantages of each method.

Key-Words: Advanced Dementia; Feeding Tubes; Malnutrition; Functional Status and Survival. 